\title{
Doenças infecciosas e parasitárias por veiculação hídrica e doenças respiratórias em área industrial, Norte do Brasil
}

\author{
Waterborne infectious and parasitic diseases and \\ respiratory diseases in an industrial area, Northern Brazil
}

\author{
Jamile Salim Marinho ${ }^{1,2}$, Iracina Maura de Jesus ${ }^{1}$, \\ Carmen Ildes Rodrigues Fróes Asmus², Marcelo de Oliveira Lima1, \\ Diomar Cavalcante Oliveira ${ }^{1,2}$
}

\begin{abstract}
Resumo
A cidade de Barcarena, no Estado do Pará, é um importante polo industrial que, nos últimos anos, esteve sujeita a registros de incidentes ambientais. Neste estudo, foi apresentado o perfil de morbidade por doenças infecciosas e parasitárias (DIP) de veiculação hídrica e por doenças respiratórias (DR) em indivíduos residentes do município de Barcarena, no período de 2008 a 2012. A pesquisa foi realizada com dados secundários sobre mortalidade e internações, obtidos a partir do Departamento de Informática do Sistema Único de Saúde (DATASUS). O coeficiente médio de mortalidade para as DIP apresentou 0,37 óbito por 1.000 habitantes. A faixa etária mais afetada pelos casos de internações por DIP foi a de 0 a 5 anos, com coeficiente médio de incidência na ordem de 31,8 por 100 mil habitantes. Para as DR, as crianças com faixa etária de 0 a 14 anos foram, aparentemente, as mais expostas, com $62,85 \%$, e os idosos acima de 60 anos, com $15,16 \%$ dos casos. As taxas de incidência e de mortalidade por DR ficaram na ordem de 39 por 100 mil habitantes. Os indicadores de saúde estudados nos alertam um risco para à saúde de populações em Barcarena e sugerem estudos quantitativos e qualitativos mais específicos de possíveis contaminações ambientais na região.

Palavras-chave: epidemiologia; doenças de veiculação hídrica; doenças respiratórias; indústrias.
\end{abstract}

\begin{abstract}
Barcarena city is an important industrial hub that has been subjected to environmental incidents in recent years. The present study reports the morbidity profile of waterborne infectious and parasitic diseases (IPD) and respiratory diseases (RD) among individuals living in Barcarena city is from 2008 to 2012. The survey was conducted with secondary data on mortality and hospital admission rates obtained from the Computer Department of the Ministry of Health - DATASUS. The average mortality rate (AM) to IPD indicated 0.37 deaths per 1000 inhabitants. The age group mostly represented in cases of hospitalizations caused by IPD was 0-5 years. The average coefficient of incidence due to this cause in the case of age group below five years of age was around 31.8/100,000 inhabitants. Regarding DR, children aged between 0 and 14 years are apparently the most exposed ones, corresponding to $62.85 \%$ of the cases, and the elderly over 60 years of age are the most exposed, with $15.16 \%$ of the cases. Incidence and mortality rates due to DR were in the order of $39 / 100,000$ inhabitants. The health indicators studied warn that populations in Barcarena are under possible risks to health and suggest that more specific studies on quantitative and qualitative possible environmental contamination in the region are still needed.
\end{abstract}

Keywords: epidemiology; waterborne diseases; respiratory diseases; industries.

\footnotetext{
${ }^{1}$ Seção de Meio Ambiente, Instituto Evandro Chagas, Secretaria de Vigilância em Saúde (SVS), Ministério da Saúde (MS) - Ananindeua (PA), Brasil. ${ }^{2}$ Instituto de Estudos de Saúde Coletiva, Universidade Federal do Rio de Janeiro (UFRJ) - Rio de Janeiro (RJ), Brasil.

Trabalho realizado no Instituto Evandro Chagas/SVS/MS - Ananindeua (PA), Brasil.

Endereço para correspondência: Jamile Salim Marinho - Seção de Meio Ambiente, Instituto Evandro Chagas, BR-316, Km 7, s/n - CEP: $67000-000$ -

Ananindeua (PA), Brasil - Email: jmilemarinho@hotmail.com

Fonte de financiamento: nenhuma.

Conflito de interesses: nada a declarar.
} 


\section{INTRODUÇÃO}

Na década de 1980, as políticas públicas brasileiras de desenvolvimento regional motivaram a implementação na Amazônia de grandes projetos de infraestrutura, exploração mineral, hidrelétricas e produção agrícola e industrial. Como consequência, houve uma rápida expansão populacional associada a processos emigratórios de indivíduos oriundos da região Nordeste do país ${ }^{1}$.

No Estado do Pará, existem vários exemplos desses grandes empreendimentos, como a construção das usinas hidrelétricas de Tucuruí, no rio Tocantins, e de Belo Monte, no rio Xingu, além da produção de alumina nas cidades de Juruti e de Barcarena.

A cidade de Barcarena teve sua economia transformada após investimentos bilaterais (Brasil e Japão) para implementação da cadeia produtiva do alumínio no Estado do Pará (produção de alumina e lingote). Incentivos fiscais atraíram outras grandes empresas, as quais se instalaram na região para a produção de ferro-gusa e o beneficiamento do caulim ${ }^{2}$. Esse rápido desenvolvimento industrial de Barcarena foi quase sempre um paradoxo entre os contínuos incrementos na produção e a ausência de mecanismos governamentais de controle dos impactos ambientais e sociais. Indivíduos atraídos, principalmente, pela disponibilidade de empregos proporcionaram um intenso e desordenado processo emigratório, o que resultou no inchaço de distritos mais antigos (Vila do Conde, Vila de Itupanema e Vila do Laranjal), construção de novos núcleos urbanos planejados (Vila dos Cabanos) e surgimento de outros conglomerados desestruturados (Vila do Canaã e Bairro Industrial), quase todos localizados às margens dos principais rios locais ${ }^{3}$.

Relatos prévios demonstram a ocorrência de alguns incidentes ambientais na região de Barcarena nos últimos anos, como os fenômenos da mortandade de peixes em igarapés ${ }^{4-6}$, o transbordamento de lama vermelha e o bloom de algas no rio Murucupi $^{5,7,8}$, e o lançamento de material particulado (fuligem) atingindo áreas urbanas 9 . Essas informações indicam que a população está exposta às mais variadas formas de contaminação (contato ou consumo de alimentos e águas contaminados ou por meio da aspiração do ar contendo material particulado oriundo dessas atividades industriais), fatores estes que podem ampliar a incidência de doenças infecciosas e parasitárias (DIP) de veiculação hídrica e doenças respiratórias (DR) ${ }^{9}$.

Essa problemática acerca de doenças causadas pela implantação de indústrias no mundo é recorrente. Um exemplo clássico aconteceu no Japão, em meados da década 1930, quando uma indústria química de fertilizantes alterou sua planta industrial e passou a utilizar sulfato de mercúrio como catalisador de sua produção, processo este que gerava o metilmercúrio como resíduo que era descartado diretamente na Baía de Minamata. Esse contaminante disponível nos compartimentos aquáticos foi incorporado à biota e, consequentemente, intoxicando milhares de pessoas que tinham como base alimentar peixes e fruto do $\operatorname{mar}^{10}$. Na Índia, na década de 1980, um grave acidente ocorreu com o isocianato de metila, quando, em uma explosão, 40 toneladas desse gás vazaram da fábrica americana de pesticidas Union Carbide. Nesse ocorrido, estimou-se que mais $10 \mathrm{mil}$ pessoas morreram devido a doenças relacionadas à inalação de gás. Atualmente, a fábrica da Union Carbide em Bhopal está abandonada, enquanto que resíduos perigosos e materiais contaminados ainda estão espalhados pela área, provocando danos aos solos e águas subterrâneas ${ }^{11-13}$.

No Brasil, temos o caso de Cubatão, no Estado de São Paulo, com a instalação de várias indústrias petroquímicas e siderúrgicas que lançaram grandes quantidades de substâncias tóxicas no meio ambiente. Em função disso, vários tipos de DR surgiram decorrentes dessa poluição industrial, assim como as DIP, associadas à ausência de infraestrutura (saneamento e habitação $)^{14}$. No Estado da Paraíba, em estudos realizados por Amaral et al. ${ }^{15}$, foi verificado também que as doenças de veiculação hídrica, como as diarreias e a dengue, estavam associadas às más condições de saneamento básico e infraestrutura, condições determinantes para a disseminação e a proliferação dessas doenças ${ }^{16}$.

Existem poucas informações e dados sistematizados sobre a incidência por DIP de veiculação hídrica e por DR em Barcarena. O estudo epidemiológico e as informações geradas neste estudo podem servir de base para delinear os efeitos da instalação dessas atividades industriais sobre o meio ambiente e suas implicações sobre a saúde e qualidade de vida da população.

\section{MATERIAS E MÉTODOS}

\section{Área de estudo}

A cidade de Barcarena pertence à mesorregião Metropolitana de Belém e à microrregião de Belém. Situa-se na interseção das coordenadas geográficas: 01³0'24” de latitude Sul e 48³7’12” de longitude a Oeste de Greenwich (Figura 1) ${ }^{17}$.

\section{Desenho do estudo}

Trata-se de um estudo descritivo com dados secundários a respeito de internações e óbitos por DIP de veiculação hídrica e por DR no município de Barcarena, no período de 2008 a 2012.

Foram obtidos dados secundários a partir do Departamento de Informática do Sistema Único de Saúde (DATASUS) sobre mortalidade e internações nos bancos de dados de Informações de Saúde - Estatísticas Vitais - Epidemiologia e Morbidade, referentes aos residentes da cidade de Barcarena, no período de 2008 a 2012. Dados populacionais, frota de veículos e saneamento básico, incluindo cobertura da rede de abastecimento de água e rede de esgoto, foram retirados do Instituto Brasileiro de Geografia e Estatística (IBGE). 


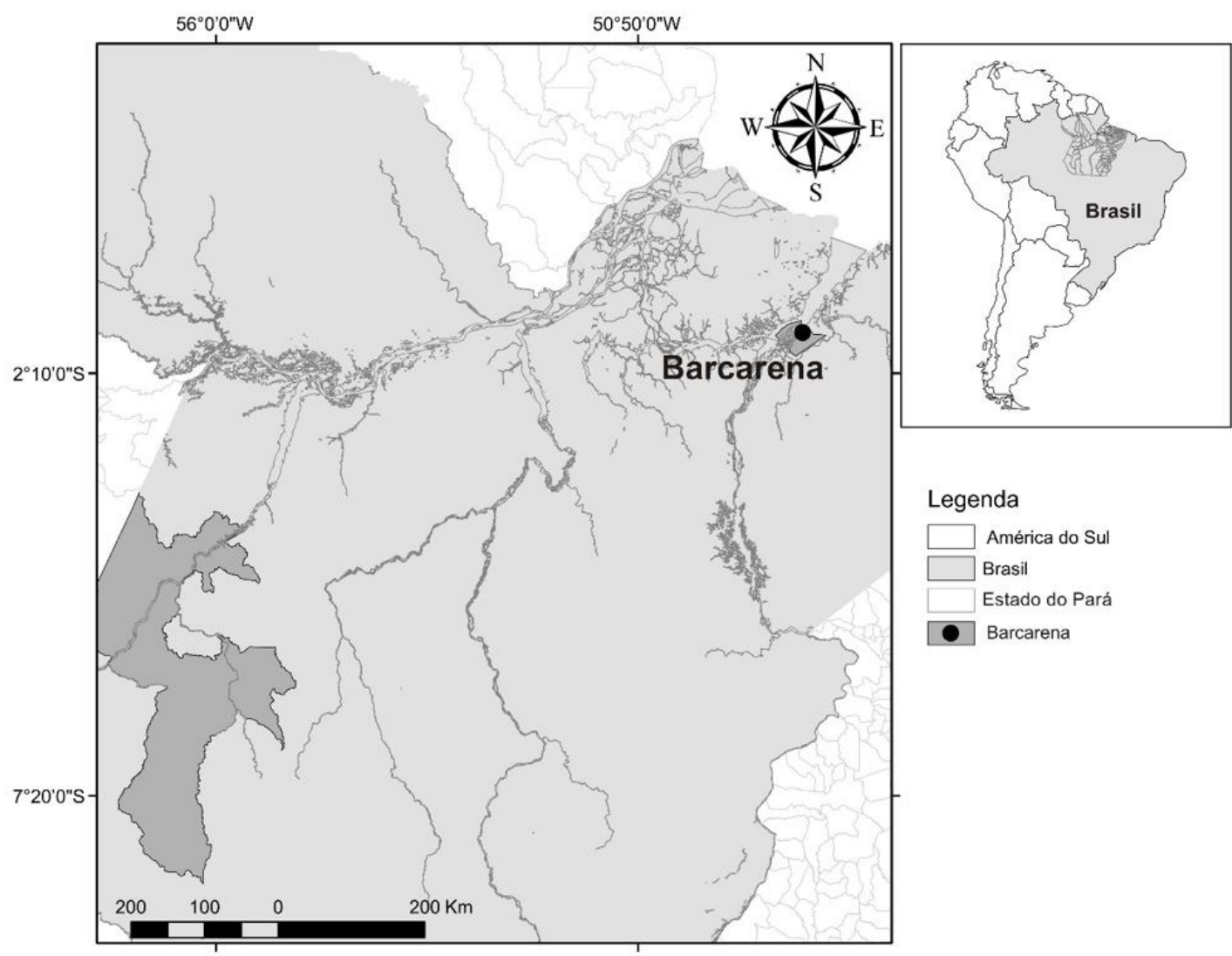

Figura 1. Mapa de localização da área de estudo. Fonte: Mapa elaborado por meio do sistema de informações do programa Terra View

\section{Critérios de inclusão}

A escolha da cidade de Barcarena foi em decorrência do crescimento populacional desordenado e do processo de industrialização que ocorreu a partir da década de 1980. Já as DR e as DIP de veiculação hídrica foram incluídas no estudo em função dos vários registros de vazamento de efluentes industriais nos principais corpos hídricos da cidade e também do crescimento da frota veicular na cidade.

\section{Estatística}

Os dados coletados do DATASUS foram armazenados e tratados por meio do aplicativo Microsoft Software Excel 2007 (planilhas eletrônicas e gráficos). No tratamento estatístico, fez-se o uso de médias aritméticas, percentagens, coeficiente de mortalidade e de incidência.

\section{RESULTADOS}

\section{Aumento populacional}

Em 1980, Barcarena era composta por 20.015 habitantes, passando para $89.909 \mathrm{em} 2008 \mathrm{e}$, com um incremento de mais 17\% nos últimos cinco anos, chegou em 2012 a um total de 105.385 habitantes. Nesse mesmo período, a população de Barcarena, em média, cresceu $4 \%$ ao ano, enquanto que, no Estado do Pará, apenas 1,66\% (Tabela 1$)^{18}$.
Tabela 1. Taxa de crescimento anual no município de Barcarena, no Estado do Pará, no período de 2008 a $2012^{16}$

Taxa de crescimento populacional anual (\%) 2008/2009 2009/2010 2010/2011 2011/2012 Média

\begin{tabular}{clllll} 
Barcarena & 2,95 & 7,87 & 2,73 & 2,64 & $\mathbf{4 , 0 4}$ \\
Pará & 1,49 & 2,01 & 1,41 & 1,73 & $\mathbf{1 , 6 6}$ \\
\hline
\end{tabular}

\section{Crescimento da frota veicular}

No período de 2007 a 2012, em Barcarena, a frota veicular aumentou de 7.443 para 16.594, equivalente a um crescimento de $122 \%$, acompanhando diretamente o aumento populacional nesses cinco anos (Figura 2).

\section{Condições de saneamento ambiental}

Em Barcarena, dados da Pesquisa Nacional de Saneamento Básico (PNSB) mostram que, em relação às condições de saneamento, apesar de terem apresentado melhorias no período de 1990-2000, ainda em 2010, 87\% da população residente (86.872 habitantes) não possuía rede geral de esgoto ou pluvial, apresentando índices elevados do uso de fossas rudimentares $(38,88 \%)$ (Tabela 2).

Ainda em 2010, o abastecimento público de água foi restrito $(28,59 \%)$, quando grande parcela da população utilizava-se ainda de outras formas de fornecimento, como poços ou 


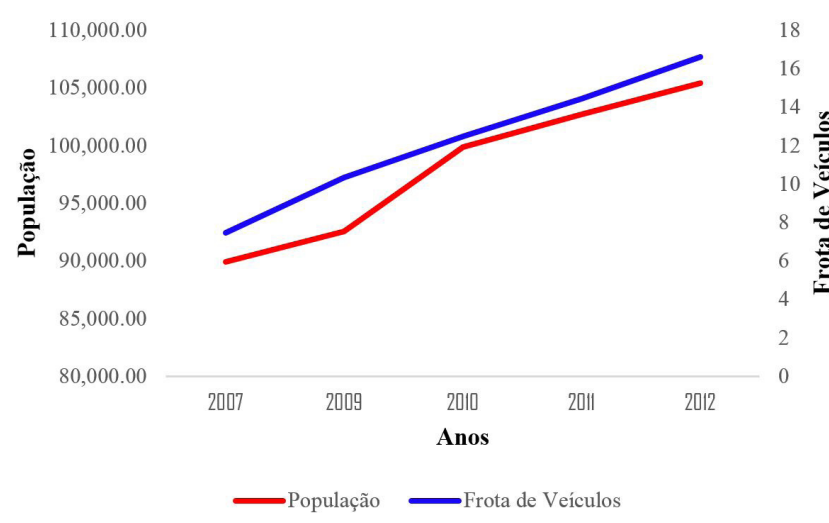

Figura 2. Comparativo do crescimento populacional com a frota veicular no município de Barcarena, no Estado do Pará, nos últimos cinco anos ${ }^{18}$

nascentes (Tabela 2). Apesar da carência no abastecimento de água, observou-se uma melhora na coleta de lixo domiciliar, atingindo mais de $67 \%$ da população ${ }^{18}$.

\section{Informações secundárias de saúde}

\section{DIP por veiculação hídrica}

Os coeficientes de mortalidade por DIP e a distribuição percentual dos casos de óbitos estão mostrados na (Tabela 3). Durante o período de 2008 a 2012, houve uma redução do número de óbitos por DIP em Barcarena. No total, foram registrados 75 casos no município nesse período. Segundo a Tabela 4, observa-se que o maior número de mortalidade por DIP ocorreu na faixa etária de 30 a 59 anos, em ambos os sexos, no período de 2008 a 2010. Nota-se também que não houve alteração nos casos na população idosa (maior que 60 anos) para ambos os sexos. Entre homens e mulheres, de um modo geral, o número de óbitos mostrou comportamento similar para todas as faixas etárias.

A análise dos coeficientes de mortalidade anual revela um coeficiente médio em todo o período estudado de 0,37 por 1.000 habitantes. O maior e o menor índice foram registrados em 2008 (0,85 por 1.000 habitantes) e em 2012 (0,18 por 1.000 habitantes), respectivamente.

As internações por DIP em Barcarena no mesmo período representaram $0,8 \%$ dos casos em todo o Estado. No Pará, houve 432.562 internações por DIP, enquanto que em Barcarena, 3.408 casos, com maior ocorrência nos anos de $2009(n=788)$ e de $2012(n=825)$.

Quanto às DIP provocadas por veiculação hídrica, é possível observar que as diarreias e as gastroenterites de origem infecciosa presumível foram as doenças que apresentaram maior frequência (91,7\%) do número total (1.244) de internações (Figura 3A). A distribuição dos casos de internações por essa causa em Barcarena entre 2008 a 2012 está estratificada por faixa etária e pode ser visualizada na Figura 3B. O maior número de casos
Tabela 2. Proporção de moradores por tipo de instalação sanitária no município de Barcarena, no Estado do Pará ${ }^{18}$

\begin{tabular}{|crcc|}
\hline Instalação sanitária & $\mathbf{1 9 9 1}$ & $\mathbf{2 0 0 0}$ & $\mathbf{2 0 1 0}$ \\
\hline Rede geral de esgoto ou pluvial & 0,1 & 12,5 & 13,08 \\
Fossa séptica & 25,8 & 25,1 & 27,21 \\
\hline Abastecimento água & $\mathbf{1 9 9 1}$ & $\mathbf{2 0 0 0}$ & $\mathbf{2 0 1 0}$ \\
\hline Rede geral & 35,1 & 26,4 & 28,59 \\
Poço ou nascente & 60,9 & 58,4 & 57,69 \\
Outra forma & 4,0 & 15,1 & 13,63 \\
\hline
\end{tabular}

Tabela 3. Número absoluto de óbitos, distribuição percentual pelo o número total de casos, coeficiente de mortalidade por 1.000 habitantes, no município de Barcarena, no Estado do Pará, 2008 a $2012^{19}$

\begin{tabular}{cccc} 
Ano & DIP & $(\%)$ & CM \\
2008 & 17 & 22,7 & 0,85 \\
2009 & 24 & 32 & 0,52 \\
2010 & 14 & 18,6 & 0,22 \\
2011 & 8 & 10,7 & 0,08 \\
2012 & 12 & 16 & 0,18 \\
Total & 75 & 100 & 0,37 \\
\hline
\end{tabular}

$\mathrm{CM}=$ Coeficiente de mortalidade, $\mathrm{DIP}=$ doenças infecciosas e parasitárias

Tabela 4. Números absolutos de óbitos por local de residência, segundo sexo e faixa etária, no município de Barcarena, no Estado do Pará, no período de 2008-2012 ${ }^{19}$

\begin{tabular}{cccccccc} 
& Faixa etária & $\mathbf{2 0 0 8}$ & $\mathbf{2 0 0 9}$ & $\mathbf{2 0 1 0}$ & $\mathbf{2 0 1 1}$ & $\mathbf{2 0 1 2}$ & Total \\
& 0 a 14 & 2 & 2 & 3 & 1 & 3 & 11 \\
\multirow{4}{*}{ Masculino } & 15 a 29 & 2 & 2 & 1 & - & 1 & 6 \\
& 30 a 59 & 5 & 5 & 4 & 1 & 2 & 17 \\
& $>60$ anos & 1 & 4 & 3 & - & 1 & 9 \\
\hline \multirow{4}{*}{ Feminino } & 0 a 14 & 2 & 2 & 1 & 1 & 2 & 8 \\
& 15 a 29 & 2 & 1 & 1 & 1 & 1 & 6 \\
& 30 a 59 & 3 & & 2 & 2 & 3 & 10 \\
\hline & 70 anos & - & 3 & 2 & 2 & 2 & 9 \\
\hline
\end{tabular}

foi observado em crianças na faixa etária de 0 a 5 anos (875), representando 70,33\%. A maior incidência ocorreu em crianças menores de 5 anos e adultos maiores de 60 anos. Para a faixa etária de 0 a 4 anos, os índices variaram entre 4,0-117,9 por 100 mil habitantes, e para o grupo acima de 60 anos, entre 11,7-20,5 por 100 mil habitantes (Figura 4).

\section{Doenças respiratórias}

O padrão de morbidade (internações) e de mortalidade por DR no município de Barcarena apresentou oscilações para o período de 2008 a 2012 (Figura 5A). As DR representaram um total de $11,8 \%(n=7.016)$ de todas as internações $(n=59.436)$ realizadas no município nesse período. Crianças na faixa etária de 0 a 14 anos e idosos acima de 60 anos foram, aparentemente, os grupos mais expostos a essas causas. No entanto, o primeiro grupo se destacou com uma variação de 53,3 a $71,7 \%$ dos casos ( $n=4.410)$, enquanto que o segundo grupo variou entre 

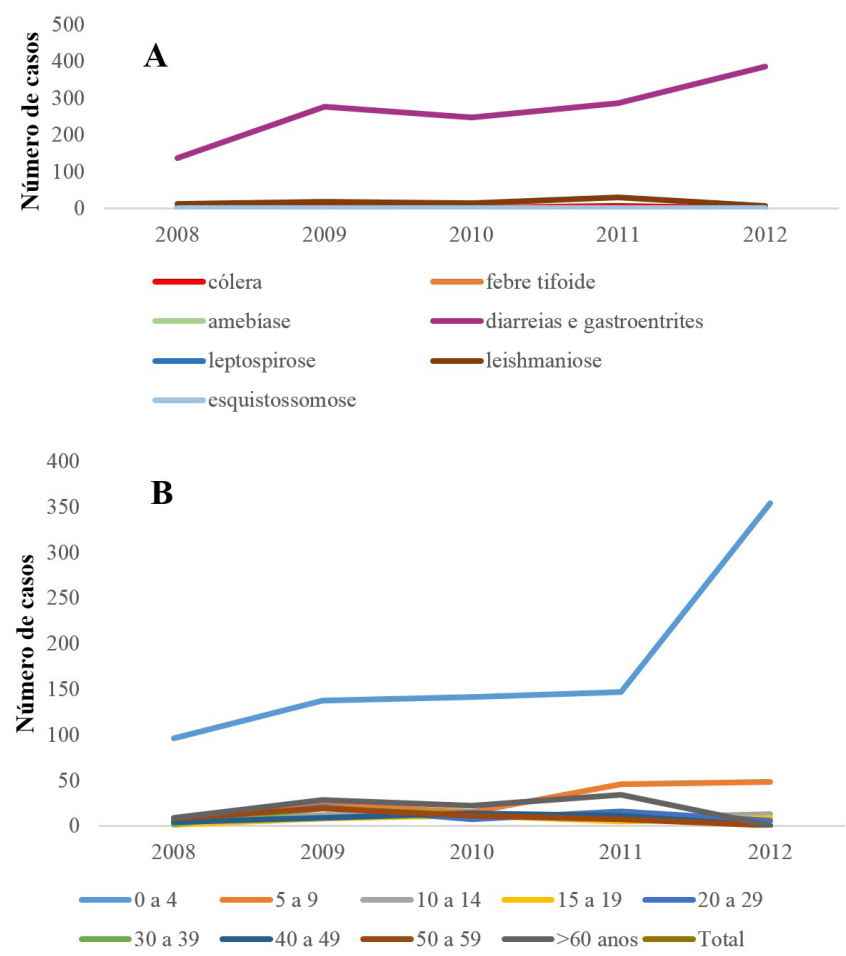

Figura 3. Distribuição do número de internações por doenças infecciosas e parasitárias (DIP) de veiculação hídrica (A) e de diarreias e gastroenterites de origem infecciosa presumível por faixa etária (B), no município de Barcarena, no Estado do Pará, no período de 2008-2012

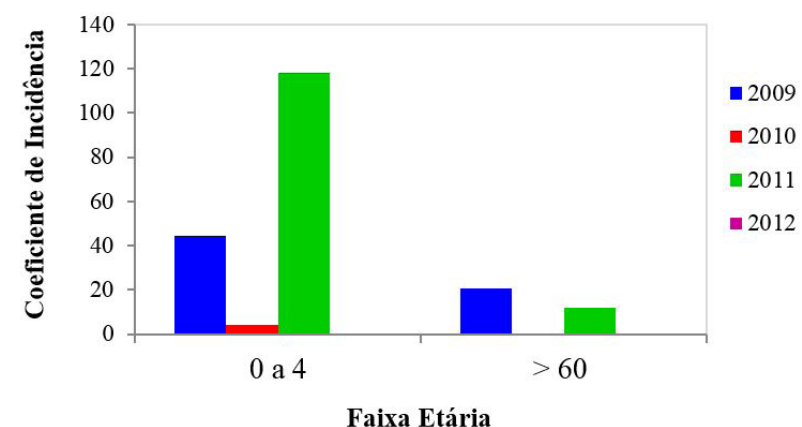

Figura 4. Distribuição por faixa etária das diarreias e gastroenterites (incidência por 100 mil habitantes), no município de Barcarena, no Estado do Pará, 2009-2012

9,7 a $22,6 \%$ dos casos $(n=1.064)$ por DR, (Figura $5 B$ ), entre os anos de 2008 a 2012.

As taxas de incidência e de mortalidade por $\mathrm{DR}$ vêm crescendo em Barcarena desde 2010. Em 2011, o coeficiente de incidência ficou na ordem de 39 por 100 mil habitantes e, em 2012, cresceu para 284,7 por 100 mil (Figura 5C).

Os casos de internações por asma em Barcarena foram mais expressivos entre as crianças, mostrando um aumento significativo de casos em cada ano, totalizando, nesse período, 469 casos em crianças de 0 a 14 anos. Já as ocorrências de notificações
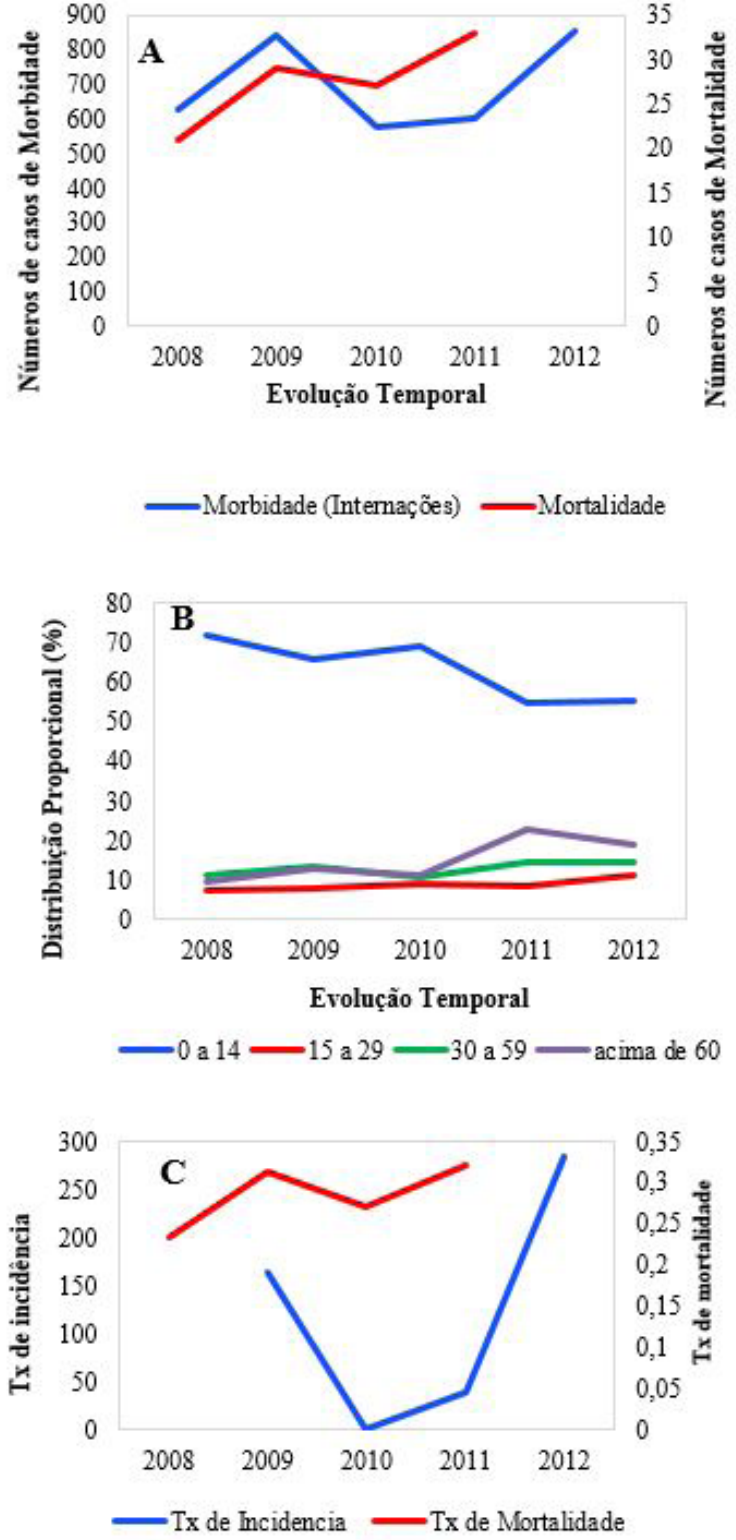

Figura 5. Perfil de morbidade e de mortalidade por doenças do aparelho respiratório $(A)$, distribuição proporcional (\%) de internações por doenças respiratórias por faixa etária $(B)$ e coeficiente de incidência vs taxa de mortalidade por doenças respiratórias (C), nos anos de 2008 a 2012, no município de Barcarena, no Estado do Pará (coeficiente de incidência 1 por 100 mil)

por DR no município, no período de 2008 a 2012, atingiram 2.827 casos, dos quais 138 pessoas vieram a óbito.

\section{DISCUSSÃO}

O aumento populacional observado durante a década de 1980 no município de Barcarena foi reportado por Coelho et al. ${ }^{20}$, que observaram um crescimento anual de 7,84\%, superior à do Estado do Pará, que, no mesmo período, foi de 3,46\%. Os autores consideraram que esse impacto na dinâmica populacional 
provavelmente estivesse associado à implantação de projetos de beneficiamento de bauxita e de caulim, além das atividades portuárias, que acarretaram mudanças sociais sobre os aspectos políticos e econômicos do desenvolvimento industrial amazônico.

Esses empreendimentos desenvolvimentistas, por disporem de atrativos, provocaram uma mudança na composição demográfica urbana do município. Segundo o IBGE, nos anos de 1970, a maioria da população economicamente ativa $(79,12 \%)$ residia no campo e desenvolvia atividades ligadas à agropecuária, extrativismo mineral e pesca. $\mathrm{O}$ aumento expressivo da população urbana nas décadas de 1980 e 1990 se deve ao fato de o município ter passado a se caracterizar como um polo industrial sediando importantes empresas ${ }^{21}$.

$\mathrm{O}$ aumento da frota veicular em Barcarena pode ser atribuído à implantação do polo industrial no município, o que, possivelmente, ocasionou a emissão de materiais poluentes para atmosfera. Gomes ${ }^{22}$ enfatiza a importância dos veículos automotores na variação interurbana das concentrações dos poluentes do ar em âmbito local. $\mathrm{O}$ autor estudou os padrões de concentração dos poluentes no entorno de vias de alto tráfego, os quais mostraram que as zonas de exposição variavam de 50 a $1.500 \mathrm{~m}$ de distância, dependendo do poluente e das condições meteorológicas.

Estudos apontam associações entre a poluição veicular e o surgimento de doenças como a asma e outros sintomas respiratórios, especialmente, em crianças ${ }^{23-26}$ e também associam essa exposição ao desenvolvimento à alergia ${ }^{24,27-29}$.

A World Health Organization (WHO $)^{30}$ define saneamento básico como o controle de todos os fatores do meio físico do homem, que exercem ou podem exercer efeitos nocivos sobre o bem-estar físico, mental e social. Pereira ${ }^{31}$ aponta a coleta e o tratamento de resíduos das atividades humanas como as principais atividades de saneamento a fim de que se preservem os recursos hídricos, garantindo, dessa forma, qualidade na água de consumo e no fornecimento para as populações, além do controle de vetores. No Brasil, é um direito assegurado pela Constituição Federal e pela Lei no 11.445/2007, que universaliza os serviços de saneamento básico, para que todos tenham acesso a esses serviços de forma adequada e de qualidade.

A exploração dos recursos naturais em Barcarena evidencia as mudanças em escala social e ambiental, as quais vêm comprometendo a qualidade dos recursos hídricos e, consequentemente, trazendo riscos para as comunidades que moram no entorno do polo industrial ${ }^{32}$, gerando conflitos socioespaciais pela introdução de novos valores na conjuntura socioeconômica local e provocando novos usos e ocupação do solo. Nesse sentido, há necessidade de infraestrutura, mais saneamento básico e mais serviços públicos.

De acordo com os dados de saneamento básico do município, é possível inferir que a população ainda está sujeita a condições de higiene inadequadas e a consumir água de má qualidade, o que favorece o surgimento de patógenos, que implicam na susceptibilidade do surgimento de doenças relacionadas com condições de saneamento básico inadequadas. Mundialmente, ao longo das últimas décadas, é evidenciada uma diminuição na mortalidade por DIP associada diretamente com as transformações sociais e econômicas (expectativa de vida ao nascer, dinâmica populacional, industrialização-urbanização e mudanças nos padrões socioculturais), que influenciaram mudanças expressivas no estilo de vida e nas relações entre pessoas ${ }^{33,34}$.

No Brasil, essa tendência também tem sido notada quando se verifica uma diminuição de 45,7 para $5,9 \%$ no período de 1930 a 1999 nos casos de mortes por DIP ${ }^{35}$. No entanto, apesar dessa redução no percentual no país, essas causas continuam em destaque, apresentando íntima ligação com condições socioeconômicas precárias (habitação, alimentação e higiene), o que reflete em baixa qualidade de vida ${ }^{36}$. No município de Barcarena, esse declínio por DIP pode estar relacionado com a melhoria no desenvolvimento do saneamento básico, apesar de ainda ser insuficiente e sujeitar a população a consumir água dos cursos de água não tratadas e, ao mesmo tempo, poluir as águas superficiais e subterrâneas com o despejo de resíduos sólidos e efluentes domésticos.

As internações por DIP em Barcarena, comparadas aos casos no Estado do Pará, podem ser reflexo do acelerado processo de industrialização-urbanização desordenado, que teve grande impacto na dinâmica populacional no município e, consequentemente, provocou aumento na produção de efluentes domésticos sem tratamento adequado, principalmente decorrente da formação de núcleos urbanos sem infraestrutura, os quais, segundo Pignatti ${ }^{37}$, são fatores que têm um impacto relevante na ocorrência desse grupo de patologias.

$\mathrm{O}$ aumento dos casos de diarreia e de gastroenterites observados no município corrobora outros estudos sobre doenças diarreicas, que confirmam uma maior incidência desses casos atingindo, principalmente, menores de 5 anos $^{38-40}$ e adultos acima de 60 anos. Essas evidências são justificadas pela vulnerabilidade desses dois grupos etários a diversos fatores (aleitamento materno, condições de habitação, saneamento básico e vacinação), permitindo concluir que quanto menor a atenção dispensada a esses grupos, maior será a incidência nessa faixa etária. Segundo Siqueira ${ }^{41}$, as diarreias são caracterizadas como doenças de grande importância dentro do quadro de saúde pública no mundo, pois são consideradas uma das principais causas de morbidade em crianças menores de 5 anos de idade, principalmente em países que não alcançaram patamares de desenvolvimento socioeconômico.

$\mathrm{O}$ aumento de casos por DR pode estar relacionado com o aumento das concentrações de poluentes atmosféricos. Essa condição é evidenciada a partir do registro de emissão de 
fuligem resultante do processo de beneficiamento de caulim para o ambiente, o que talvez esteja prejudicando a qualidade do ar da região e, dessa forma, levando ao aumento de casos de DR no município ${ }^{42}$.

As crianças e os idosos são considerados grupos de risco em relação à poluição atmosférica, uma vez que a absorção de agentes químicos pelas vias áreas pode estar relacionada com características peculiares, as quais tornam esses grupos mais vulneráveis. Gonçalves et al. ${ }^{43}$ citam alguns fatores que contribuem para tornar esses grupos mais vulneráveis às DR: para as crianças, velocidade de crescimento, maior área de perda de calor por unidade de peso, elevadas taxas de metabolismo em repouso e consumo de oxigênio; para os idosos, a baixa imunidade e a redução da função ciliar. Estudos relatam que os primeiros dois anos de vida são um período crítico para o desenvolvimento da asma e que existem evidências de que a interação entre a carga genética e a exposição a poluentes ambientais permite que as crianças sejam mais suscetíveis a tornarem-se atópicas ${ }^{44}$.

Segundo a $\mathrm{WHO}^{30}$, a ocorrência de asma pode estar associada a alguns poluentes (fumaça do tabaco, dióxidos de nitrogênio e de enxofre, material particulado e ozônio), tanto no ambiente interno quanto externo, e a presença de poeira, mofo, ácaros e umidade no ambiente interno. McConnell et al. ${ }^{45}$ fizeram um estudo em crianças com asma em algumas comunidades na Califórnia, nos Estados Unidos, relacionando os sintomas da bronquite com poluentes como o carbono orgânico, material particulado e dióxido de nitrogênio, e verificaram associações positivas.

As ocorrências de notificações por DR em Barcarena no período estudado pode estar diretamente relacionado à poluição atmosférica, uma vez que estudos realizados em Jerusalém e na China observaram associações significativas entres os poluentes atmosféricos (material particulado e dióxidos de nitrogênio e de enxofre) com internações hospitalares por $\mathrm{DR}^{46,47}$. Seguindo uma tendência mundial, no Brasil, mais especificamente em Minas Gerais, no Paraná e em São Paulo, pesquisas feitas por Bueno et al. ${ }^{48}$, Bakonyi et al. ${ }^{49}$, Freitas et al. ${ }^{50}$ e Nascimento ${ }^{51}$ mostraram também associação entre a concentração de poluentes atmosféricos e o incremento na morbidade por problemas respiratórios, por meio de correlações lineares positivas sobre a poluição do ar e as internações por DR.

\section{CONCLUSÃO}

A implantação do complexo industrial na década de 1980, além de estimular o crescimento populacional em Barcarena, trouxe reflexos ao meio ambiente que podem sugerir implicações na saúde da população.

As notificações de mortalidade e de morbidade (internações) por DIP de veiculação hídrica e por DR em Barcarena oscilaram ao longo dos cinco anos estudados e demonstraram que as crianças são o principal grupo de risco no município.

$\mathrm{O}$ aumento das taxas de incidência e mortalidade por DR em Barcarena pode ser um reflexo do aumento da frota veicular, assim como das emissões de poluentes industriais ocorridos no município.

$\mathrm{O}$ aumento dos casos de diarreias e de gastroenterites de origem infecciosa presumível no município de Barcarena talvez esteja relacionado com as condições de saneamento básico, apesar de esses serviços terem apresentado avanços discretos nas áreas de abastecimento de água e instalação sanitária.

Os indicadores de saúde estudados nos alertam para possíveis implicações na saúde da população, havendo a necessidade de estudos quantitativos e qualitativos mais específicos, sobre a qualidade do ar e das águas no município e que possam contribuir para a melhoria da vigilância em saúde ambiental na região.

\section{REFERÊNCIAS}

1. Becker B. Amazônia: geopolítica na virada do III Milênio. 2. ed. Rio de Janeiro: Garamond; 2007.

2. Magno A, Barbosa S. A naturalização da identidade social preconizada na indústria do alumínio primário paraense. Sociologias. 2010;12(23):268-303.

3. Coelho MCN, Monteiro MA, Santos IC. Políticas públicas, corredores de exportação, modernização portuária, industrialização e impactos territoriais e ambientais no município de Barcarena, Pará. NAEA. 2004;11(1):141-78.

4. Santos ECO, Brabo ES, Sá LLC, Lima MO, Girard RPM. Relatório técnico da avaliação da mortandade de peixes no Rio Murucupi ocorrida no dia 04/04/03, no município de Barcarena, Estado do Pará. Ananindeua: Instituto Evandro Chagas; 2013 [citado em 2013 mar 1]. Disponível em: www.iec. pa.gov.br

5. Brabo ES, Lima MO, Santos JG, Jesus IM, Ferreira HS. Avaliação técnica sobre o fenômeno da mortandade de peixes ocorrido no dia 23/06/2003 na praia de Itupanema no município de Barcarena, Estado do Pará. Ananindeua: Instituto Evandro Chagas; 2013 [citado em 2013 mar 1]. Disponível em: www.iec.pa.gov.br

6. Carneiro BS, Faial KRF, Lima MO, Ferreira FSF. Caracterização Físicoquímica dos Igarapés Curuperê e Dendê, localizados no Município de Barcarena, Pará. Ananindeua: Instituto Evandro Chagas; 2013 [citado em 2013 mar 1]. Disponível em: www.iec.pa.gov.br

7. Sá LLC, Mendes RA, Mendonça NM. Relatório IEC-SAMAM 002/2006: ocorrência de um Bloom de Algas nos Rios e Igarapés do Município de Barcarena-Pará. Ananindeua: Instituto Evandro Chagas; 2011 [citado em 2011 mar 1]. Disponível em: www.iec.pa.gov.br

8. Lima MO, Brabo ES. Relatório Técnico dos Impactos sobre os Igarapés Curuperê e Dendê em Decorrência do Lançamento de Efluentes da Empresa Imerys Rio Capim Caulim (IRCC) no município de Barcarena, Estado do Pará. Ananindeua: Instituto Evandro Chagas; 2011 [citado em 2011 mar 1]. Disponível em: www.iec.pa.gov.br 
9. Craun GF, Calderon RL, Wade TJ. Assessing waterborne risks: an introduction. J Water Health. 2006;4(Supl 2):3-18. PMid:16895083. http:// dx.doi.org/10.2166/wh.2006.015.

10. Environmental Health Department. Our intensive efforts to overcome the tragic history of Minamata Disease. Tokyo: Environmental Agency of Japan; 1999. In Japanese.

11. Sharan M, McNider RT, Gopalakrishnan SG, Singh MP, Gopalakrishnan SG. Bhopal vazamento de gás: a simulação numérica de dispersão episódica. Atmos Environ. 1994; 29(16):2061-74. http://dx.doi.org/10.1016/13522310(95)00031-S.

12. Edwards DW. Export inherent safety NOT risk. J Loss Prev Process Ind. 2005;18(4-6):254-60. http://dx.doi.org/10.1016/j.jp.2005.06.014.

13. Eckerman I. The Bhopal gas leak: analyses of causes and consequences by three different models. J Loss Prev Process Ind. 2005;18(4-6):213-7. http:// dx.doi.org/10.1016/j.jlp.2005.07.007.

14. Guilherme ML. Urbanização, saúde e meio ambiente: o caso da implantação do pólo industrial de Cubatão e seus efeitos urbanos e regionais nos setores da saúde e poluição ambiental. In Taratalia JC, Oliveira OL, editores. Modernização no interior de São Paulo. São Paulo: UNESP; 1988.

15. Amaral LA, Nader Fo A, Rossi Jr OD, Ferreira FL, Barros LS.. Água de consumo humano como fator de risco a saúde em propriedades rurais. Rev Saude Publica. 2003;37(4):510-4. PMid:12937713. http://dx.doi.org/10.1590/ S0034-89102003000400017.

16. Franco RMB. Protozoários de Veiculação hídrica: relevância em saúde pública. Rev. Panam. Infectol. 2007;9(1):36-43.

17. Martins SEM, Mendes AC. Caracterização de depósitos sedimentares recentes da porção superior da Baía de Marajó (margem leste do estuário do Rio Pará, Amazônia). Pesqui. Geocienc. 2011;38(2):168-80.

18. Instituto Brasileiro de Geografia e Estatística. Cidades. Rio de Janeiro; 2014 [citado em 2014 mar 30]. Disponível em: http://www.cidades.ibge.gov.br/ xtras/home.php

19. Brasil. Portal da Saúde. DATASUS: Sistema de Informações Hospitalares do SUS. Morbidade Hospitalar do SUS por local de residência. Brasília; 2014 [citado em 2014 maio 1]. Disponível em: http://tabnet.datasus.gov. br

20. Coelho MCN, Monteiro MA, Lira SRB, Lopes AG. Estratégias de modernização na Amazônia e a (re)estruturação de municípios: o caso da implantação de empresas minero metalúrgicas e de energia elétrica. In: Gonçalves MF, Brandão CA, Galvão AC, editores. Regiões e cidades, cidades nas regiões: o desafio urbano-regional. 1. ed. São Paulo: UNESP; 2003. p. 657-94. vol. 1.

21. Coelho MCN, Monteiro MA, Santos IC. Políticas públicas, corredores de exportação, modernização portuária, industrialização e impactos territoriais e ambientais no município de Barcarena, Pará. NAEA. 2004;11(1):141-78.

22. Gomes MJM. Ambiente e pulmão. J. Pneumol. 2002;28(5):261-9. http:// dx.doi.org/10.1590/S0102-35862002000500004.

23. Ko FW, Hui DS. Air pollution and chronic obstructive pulmonary disease. Respirology. 2012;17(3):395-401. PMid:22142380.

24. Brauer M, Hoek G, Smit HA, Jongste JC, Gerritsen J, Postma DS, et al. Air pollution and development of asthma, allergy and infections in a birth cohort. Eur Respir J. 2007;29:879-88. PMid:17251230. http://dx.doi. org/10.1183/09031936.00083406.
25. Jerrett M, Shankardass K, Berhane K, Gauderman WJ, Künzli N, Avol E, et al. Traffic-related air pollution and asthma on set in children: a prospective cohort study with individual exposure measurement. Environ Health Perspect. 2008;116(10):1433-8. PMid:18941591. http://dx.doi.org/10.1289/ ehp.10968.

26. Kim JJ, Huen K, Adams S, Smorodinsky S, Hoats A, Malig B, et al. Residential traffic and children's respiratory health. Environ Health Perspect. 2008;116(9):1274-9. PMid:18795175.

27. Brauer M, Gehring U, Brunekreef B, Jongste J, Gerritsen J, Rovers M, et al. Traffic-related air pollution and otitis media. Environ Health Perspect. 2006;114(9):1414-8. PMid:16966098.

28. Cesaroni G, Badaloni C, Porta D, Forastiere F, Perucci CA. Comparison between various sindices of exposure to traffic related air pollution and their impact on respiratory health in adults. Occup Environ Med. 2008;65(10):68390. PMid:18203803. http://dx.doi.org/10.1136/oem.2007.037846.

29. Morgenstern V, Zutavern A, Cyrys J, Brockow I, Koletzko S, Krämer U, et al. Atopic diseases, allergic sensitization, and exposure to traffic related air pollution in children. Am J Respir Crit Care Med. 2008;177(12):1331-7. PMid:18337595. http://dx.doi.org/10.1164/rccm.200701-036OC.

30. World Health Organization. Sanitation. Geneva; 2014 [citado em 2014 jun 1]. Disponivel em: http://www.who.int/mediacentre/factsheets/fs392/en/

31. Pereira DSP. Saneamento básico: situação atual na América Latina: enfoque Brasil. España; 2002 [citado em 2014 jul 10]. Disponível em: http://tierra. rediris.es/hidroded/RVA.html

32. Lima MO, Santos ECO, Jesus IM, Medeiros AC, Faial KCF, Alves CN. Assessment of surface water in two amazonian rivers impacted by industrial wastewater, Barcarena City, Pará State (Brazil). J Braz Chem Soc. 2011;22(8):1493-504. http://dx.doi.org/10.1590/S0103-50532011000800013.

33. Frenk J, Bobadilla JL, Sepuúlveda J, Cervantes ML. Health transition in middle-income countries: new challenges for health care. Health Policy Plan. 1989 v;4(1):29-39. http://dx.doi.org/10.1093/heapol/4.1.29.

34. Bobadilha JL, Frenk J, Lozano R, Freijka T, Stern C. The epidemiologic transition and health priorities. In: Jamison DT. Diseases control priorities in developing countries. Oxford: Oxford University Press; 1993. p. 161-87. (Oxford Medical Publications).

35. Carmo EH, Maurício LB, Silva Jr JB. Mudanças nos padrões de morbimortalidade da população brasileira: os desafios para um novo século. Epidemiol. Serv. Saude. 2003;12(2):63-75.

36. Brasil. Ministério da Saúde. Secretaria de Vigilância em Saúde. Departamento de Análise de Situação de Saúde. Saúde Brasil 2013: uma análise da situação de saúde e das doenças transmissíveis relacionadas a pobreza. Brasília; 2014.

37. Pignatti MG. Saúde e Ambiente: as doenças emergentes no Brasil. Ambient. Soc. 2004;7(1):133-47. http://dx.doi.org/10.1590/S1414-753X2004000100008.

38. Waldman EA, Barata RC, Moraes JC, Guibu IA, Timenetsky MC. Gastroenterites e infecções respiratórias agudas em crianças menores de 5 anos, em área da região Sudeste do Brasil, 1986-1987. II - Diarréias. Rev Saude Publica. 1997;31(1):62-70. PMid:9430927. http://dx.doi.org/10.1590/ S0034-89101997000100009.

39. Paes NA, Silva LAA. Doenças infecciosas e parasitárias no Brasil: uma década de transição. Am J Public Health. 1999;6(2):99-109.

40. Araújo MFM, Ferreira AB, Gondim KM, Chaves ES. A prevalência de diarréia em crianças não amamentadas ou com amamentação por tempo inferior a seis meses. Ciencia Cuid. Saúde. 2007;6(1):76-84. 
41. Siqueira AA. Surto de gastroenterite por rotavírus no município de Rio Branco - AC. Bol. Eletr. Epidem. 2006;6(8):2-6.

42. Faial KF, Mendes RA, Rocha CCS, Lopes ASC, Alves FAS, Souza ML, et al. Relatório Técnico do Impacto sobre a comunidade do Bairro Industrial em decorrência do lançamento de Material Particulado (Fuligem) da empresa Imerys Rio Capim Caulim (IRCC) no Município de Barcarena, Estado do Pará. Ananindeua: Instituto Evandro Chagas; 2014 [citado em 2014 mar 20]. Disponível em: www.iec.pa.gov.br

43. Gonçalves K, Castro HA, Hacon SS. As queimadas na região amazônica e o adoecimento respiratório. Cien Saude Colet. 2012;17(6):1523-32. PMid:22699643. http://dx.doi.org/10.1590/S1413-81232012000600016.

44. Zmirou D, Gauvin S, Pin I, Momas I, Sahraoui F, Just J, et al. Traffic related air pollution and incidence of childhood'sasthma: results of theVesta case controlstudy. J Epidemiol Community Health. 2004;58(1):18-23. PMid:14684722. http://dx.doi.org/10.1136/jech.58.1.18.

45. McConnell R, Berhane K, Gilliland F, Molitor J, Thomas D, Lurmann F, et al. Prospective study of air pollution and bronchitic symptoms in children with Asthma. Am J Respir Crit Care Med. 2003;168(7):790-7. PMid:12893648. http://dx.doi.org/10.1164/rccm.200304-466OC.

46. Tao Y, Mi S, Zhou S, Wang S, Xie X. Air pollution and hospital admissions for respiratory diseases in Lanzhou, China. Environ Pollut. 2014;185:196201. PMid:24286694. http://dx.doi.org/10.1016/j.envpol.2013.10.035.
47. Nirel R, Schiff M, Paltiel O. Internações respiratórias em crianças e exposição residencial a poluição do ar tráfego em Jerusalém. J. Inter. Hig. S. Amb. 2015;218(1):34-40.

48. Bueno FF, Fonseca AR, Braga FA, Miranda PSC. Qualidade do ar e internações por doenças respiratórias em crianças no município de Divinópolis, Estado de Minas Gerais. Acta. Sci. Health. Sci. 2010;32(2):185-9.

49. Bakonyi SMC, Danni-Oliveira IM, Martins LC, Braga ALF. Poluição atmosférica e doenças respiratórias em crianças na cidade de Curitiba, PR. Rev Saude Publica. 2004;38(5):695-700. PMid:15499441. http://dx.doi. org/10.1590/S0034-89102004000500012.

50. Freitas C, Bremner SA, Gouveia N, Pereira LAA, Saldiva PHN. Internações e óbitos e sua relação com a poluição atmosférica em São Paulo, 1993 a 1997. Rev Saude Publica. 2004;38(6):751-7. PMid:15608891. http://dx.doi. org/10.1590/S0034-89102004000600001.

51. Nascimento LFC, Pereira LA, Braga AL, Módolo MC, Carvalho Jr JA. Efeitos da poluição atmosférica na saúde infantil em São José dos Campos, SP. Rev Saude Publica. 2006;40(1):77-82. PMid:16410986. http://dx.doi. org/10.1590/S0034-89102006000100013.

Recebido em: Maio 06, 2016 Aprovado em: Ago. 29, 2016 